\title{
Maximal families of nodal varieties with defect
}

\author{
Remke Kloosterman ${ }^{1}$
}

Received: 2 March 2020 / Accepted: 1 July 2021 / Published online: 30 July 2021

(c) The Author(s) 2021

\begin{abstract}
In this paper we prove that a nodal hypersurface in $\mathbf{P}^{4}$ with positive defect has at least $(d-1)^{2}$ nodes, and if it has at most $2(d-2)(d-1)$ nodes and $d \geq 7$ then it contains either a plane or a quadric surface. Furthermore, we prove that a nodal double cover of $\mathbf{P}^{3}$ ramified along a surface of degree $2 d$ with positive defect has at least $d(2 d-1)$ nodes. We construct the largest dimensional family of nodal degree $d$ hypersurfaces in $\mathbf{P}^{2 n+2}$ with positive defect for $d$ sufficiently large.
\end{abstract}

Keywords Nodal varieties with positive defect · Noether-Lefschetz theory

Mathematics Subject Classification $32 \mathrm{~S} 20 \cdot 14 \mathrm{~J} 30 \cdot 14 \mathrm{~J} 70 \cdot 14 \mathrm{M} 10$

\section{Introduction}

Let $n \geq 3$ be an odd integer and $c$ be a positive integer. Let $1 \leq w_{0} \leq \cdots \leq w_{n+c}$ and $2 \leq$ $d_{1} \leq \cdots \leq d_{c}$ be integers. For a nodal complete intersection $X \subset \mathbf{P}\left(w_{0}, \ldots, w_{n+c}\right)=$ : $\mathbf{P}$ of multidegree $d_{1}, \ldots, d_{c}$ we define the defect of $X$ to be $h^{n+1}(X)-h^{n-1}(X)$. In this paper we consider the problem of determining the minimal number of nodes to have positive defect. With a node we intend an isolated ordinary double point. In this generality the problem is too hard.

In the sequel we concentrate on the two special cases: hypersurfaces in $\mathbf{P}^{n+1}$ and double solids, i.e., hypersurfaces of degree $2 k$ in $\mathbf{P}(k, 1,1,1,1)$. In subsequent papers we will discuss the case of three-dimensional complete intersections in $\mathbf{P}^{3+c}$ and of elliptic threefolds over $\mathbf{P}^{2}$, i.e., hypersurfaces of degree $6 k$ in $\mathbf{P}(2 k, 3 k, 1,1,1)$.

We start by recalling some previous results on this problem. For hypersurfaces of degree $d \geq 2$ in $\mathbf{P}^{4}$, Cheltsov showed that the minimal number of nodes to have positive defect is

The author thanks Ivan Cheltsov, Slawomir Cynk, Vincenzo Di Gennaro, Brendan Hassett and Orsola Tommasi for several comments on a previous version of this paper. The author would like to thank the referees for several suggestions to improve the presentation. The author is partially supported by DFG-grant KL 2244/2-1.

$凶$ Remke Kloosterman

klooster@math.unipd.it

1 Dipartimento di Matematica, Università degli Studi di Padova, Via Trieste 63, 35121 Padova, Italy 
$(d-1)^{2}[2]$ and that if $X$ has positive defect and $(d-1)^{2}$ nodes then $X$ contains a plane [3]. This improves a previously known bound by Ciliberto and Di Gennaro [5]. Ciliberto and Di Gennaro showed that if a hypersurface with positive defect has at most $2(d-2)(d-1)$ nodes and the defect is caused by a smooth surface, then $X$ contains either a plane or a quadric surface.

To illustrate our methods we start by giving a new proof of Cheltsov's theorem

Theorem 1.1 (Cheltsov [2,3]) Let $X \subset \mathbf{P}^{4}$ be a nodal hypersurface of degree $d$, with $d \geq 3$. Assume that $h^{4}(X) \geq 2$. Then $X$ has at least $(d-1)^{2}$ nodes. Moreover, if equality holds then $X$ contains a plane.

We included this proof because it is a good illustration of our techniques and is significantly different from Cheltsov's original proof.

For fixed integers $n$ and $d$ let $\mathrm{DEF}_{d} \subset \mathbf{C}\left[x_{0}, \ldots, x_{2 n+2}\right]_{d}$ be the locus of polynomials $f$ such that $V(f)$ is a nodal hypersurface with positive defect.

Theorem 1.2 Fix $n \in \mathbf{N}, n \geq 2$. Then there exists a $D$ such that

$$
\operatorname{codim} L \geq\left(\begin{array}{c}
d+n+1 \\
n+1
\end{array}\right)-(n+1)(n+2)
$$

holds for every $d>D$ and every irreducible component $L$ of $\mathrm{DEF}_{d}$.

Moreover, if $n=1$ or Conjecture 1 of [17] holds then we can take $D=2$ and any hypersurface in $\mathrm{DEF}_{d}$ has at least $(d-1)^{n+1}$ nodes.

Otwinowska showed that [17, Conjecture 1] is implied by the Conjecture of Eisenbud, Green and Harris on the Hilbert functions of an ideal containing a complete intersection ideal.

We also consider the case of double covers of $\mathbf{P}^{3}$. In this case we recover a result by Cheltsov:

Theorem 1.3 (Cheltsov [1]) Let $f \in \mathbf{C}\left[x_{0}, x_{1}, x_{2}, x_{3}\right]$ be a homogeneous polynomial of degree $2 d$ such that $V(f)$ is a nodal surface. Let $X: y^{2}=f$ be the double cover branched along $V(f)$. Suppose $h^{4}(X)>1$. Then $X$ has at least $d(2 d-1)$ nodes.

In the case of hypersurfaces of degree $d$ in $\mathbf{P}^{4}$ we prove the Ciliberto-Di Gennaro conjecture for $d \geq 7$ :

Theorem 1.4 Let $X \subset \mathbf{P}^{4}$ be a nodal hypersurface of degree $d$, with d at least 7. Suppose that $X$ is non-factorial and that $X$ has at most $2(d-2)(d-1)$ nodes then either $X$ contains a plane and has $(d-1)^{2}$ nodes or $X$ contains a quadric surface and has exactly $2(d-1)(d-2)$ nodes.

This conjecture has been proved for $d=3$ by [9] and for $d=4$ by [4,20]. Hong considers the nonfactoriality in the case of $d=4$ and 13 nodes, see [14]. The results of Cheltsov mentioned above imply this result for up to $(d-1)^{2}$ nodes.

We will now briefly discuss the strategy of proof. To reprove Cheltsov's result we use the following strategy: Let $I$ be the ideal of the nodes of $X$, where $X$ is a nodal hypersurface of degree $d$ with positive defect. Let $H=V(\ell)$ be a general hyperplane then $X \cap H$ is smooth. In particular the ideal $I_{H}:=(I, \ell)$ defines an empty scheme. Since $X$ has positive defect this implies that the Hilbert polynomial of $I$ and the Hilbert function of $I$ are different in degree $2 d-5$. From this it follows that $h_{I_{H}}(2 d-4) \neq 0$ holds. Since the partials of the defining equation for $X$ are contained in $I_{d-1}$, it follows that $I_{d-1}$ has finitely many base points. Therefore $I_{d-1, H}$ is base point free. A combination of results from Macaulay and 
Gotzmann on which functions occur as Hilbert functions of ideals yields that $h_{I_{H}}(k) \geq k+1$ for $k \leq d-2$ and that $h_{I_{H}}(k) \geq 2 d-3-k$, for $d-1 \leq k \leq 2 d-3$. The observation $p_{I} \geq h_{I}(2 d-4) \geq \sum_{k=0}^{2 d-4} h_{I_{H}}(k)$ finishes the proof. The ideal $I_{H}$ is very similar to the ideal used by Green [12] to determine the largest component of the Noether-Lefschetz locus of surfaces in $\mathbf{P}^{3}$.

The other proofs are variations of this idea. In the case of an hypersurface in $\mathbf{P}^{2 n+2}$ with $n \geq 2$, Macaulay's result is not strong enough to obtain the desired lower bound. In this case we use a result by Otwinowska [17] instead. However this result bounds $h_{I_{H}}(k)$ only in a certain interval. This is sufficient to detect the largest dimensional component, but not to establish the minimal number of nodes. If one assumes a conjecture from [17], then one obtains the desired lower bound for the number of nodes. The double solid case is very similar to the case of hypersurfaces in $\mathbf{P}^{4}$ and we will not comment on this.

The paper is organized as follows. In Sect. 2 we recall several standard results on the Hilbert functions of ideals. In Sect. 3 we recall some standard results on the cohomology of nodal complete intersections. In particular, we present a formula to calculate the defect of a nodal hypersurface. In Sect. 4 we prove the results for the hypersurfaces and in the double covers, except for the Ciliberto-Di Gennaro conjecture, which is proven in Sect. 5.

\section{Macaulay's and green's result}

Let $S=\mathbf{C}\left[x_{0}, \ldots, x_{n}\right]$ and let $I \subset S$ be a homogeneous ideal. Let $h_{I}$ be the Hilbert function of $I$, i.e., $h_{I}(k)=\operatorname{dim}(S / I)_{k}$. Let $p_{I}(t) \in \mathbf{Q}[t]$ be the Hilbert polynomial, i.e., the polynomial such that $h_{I}(k)=p_{I}(k)$ for $k \in \mathbf{Z}, k$ sufficiently large.

Let $d \geq 1$ be an integer. Let $c:=h_{I}(d)$. We can write $c$ uniquely as

$$
c=\sum_{i=1}^{d}\left(\begin{array}{c}
i+\epsilon_{i} \\
i
\end{array}\right)
$$

with $\epsilon_{d} \geq \epsilon_{d-1} \geq \ldots \geq \epsilon_{1} \geq-1$. We call this the (Macaulay) expansion of $c$ in base $d$. This expansion can be obtained inductively as follows: The number $\epsilon_{d}$ is the largest integer such that $\left(\begin{array}{c}d+\epsilon_{d} \\ d\end{array}\right) \leq c$. The numbers $\epsilon_{i}$ for $i<d$ are the coefficients in the expansion of $c-\left(\begin{array}{c}d+\epsilon_{d} \\ d\end{array}\right)$ in base $d-1$.

Using the Macaulay expansion of $c$ we define the following numbers:

$$
c^{\langle d\rangle}:=\sum_{i=1}^{d}\left(\begin{array}{c}
i+\epsilon_{i}+1 \\
i+1
\end{array}\right), c_{\langle d\rangle}:=\sum_{i=1}^{d}\left(\begin{array}{c}
i+\epsilon_{i}-1 \\
i
\end{array}\right), c_{* d}:=\sum_{i=2}^{d}\left(\begin{array}{c}
i+\epsilon_{i}-1 \\
i-1
\end{array}\right) .
$$

Note that $c \mapsto c_{* d}, c \mapsto c^{\langle d\rangle}$ and $c \mapsto c_{\langle d\rangle}$ are increasing functions in $c$.

Recall the following theorem by Macaulay:

Theorem 2.1 (Macaulay [16]) Let $V \subset S_{d}$ be a linear system and $c=\operatorname{codim} V$. Then the codimension of $V \otimes_{\mathbf{C}} S_{1}$ in $S_{d+1}$ is at most $c^{\langle d\rangle}$.

We apply this result mostly in the case where $V$ is the degree- $d$ part of an ideal $I$. In this case we can also obtain information on $h_{I}(d-1)$.

Corollary 2.2 Let $I \subset S$ be an ideal, $d \geq 2$ an integer and $c:=h_{I}(d)$. Then

$$
h_{I}(d-1) \geq c_{* d} .
$$

Moreover, if $\epsilon_{1}$ is nonnegative then $h_{I}(d-1)>c_{* d}$ holds. 
For small $c$ we have the following Macaulay expansions in base $d$ :

- For $c \leq d$ we have $\epsilon_{d}=\cdots=\epsilon_{d-c+1}=0$ and $\epsilon_{d-c}=\cdots=\epsilon_{1}=-1$. Hence $c^{\langle d\rangle}=c$.

- For $d+1 \leq c \leq 2 d$ we have $\epsilon_{d}=1, \epsilon_{d-1}=\cdots=\epsilon_{d-a}=0, \epsilon_{d-a-1}=\cdots=\epsilon_{1}=-1$, where $a=c-d-1$. Hence $c^{\langle d\rangle}=c+1$.

- For $c=2 d+1$ we have $\epsilon_{d}=\epsilon_{d-1}=1$ and all other $\epsilon_{i}$ equal -1 . Hence $c^{\langle d\rangle}=2 d+3=$ $c+2$.

Applying the previous corollary repeatedly yields

Corollary 2.3 Let $I \subset S$ be an ideal, $d \geq 2$ an integer and $c:=h_{I}(d)$. For $0 \leq k \leq d$ we have that

$$
h_{I}(k) \geq \begin{cases}\min (c, k+1) & \text { if } c \leq d \\ \min (k+(c-d), 2 k+1) & \text { if } d+1 \leq c \leq 2 d \\ 2 k+1 & \text { if } c=2 d+1\end{cases}
$$

The following result will be used to detect the Hilbert polynomial of the ideal generated by $I_{d}$ :

Theorem 2.4 (Gotzmann [10]) Let $V \subset S_{d}$ be a linear system and let $J \subset S$ be the ideal generated by $V$. Set $c=h_{J}(d)$. If $h_{J}(d+1)=c^{\langle d\rangle}$ then for all $k \geq d$ we have $h_{J}(k+1)=$ $h_{J}(k)^{\langle k\rangle}$. In particular the Hilbert polynomial $p_{J}(t)$ of $J$ is given by

$$
\sum_{i=1}^{d}\left(\begin{array}{c}
t+\epsilon_{i} \\
t
\end{array}\right)
$$

and the dimension of $V(J)$ equals $\epsilon_{d}$.

We use this result mostly in the case where $c \leq d$ :

Corollary 2.5 Let $I \subset S$ be an ideal such that $h_{I}(d) \leq d$ and $I_{d+1}$ is base point free. Then for all $k \geq d$ we have $h_{I}(k+1)<h_{I}(k)$ or $h_{I}(k)=0$.

Proof If $h_{I}(d)=0$ then $h_{I}(k)=0$ for all $k \geq d$ and we are done. Suppose now that $h_{I}(d)>0$.

We prove the result by induction. Hence it suffices to show that $0<h_{I}(d)<d$ implies that $h_{I}(d+1)<h_{I}(d)$.

Let $I^{\prime}$ be the ideal generated by $I_{d}$. From the fact that $h_{I^{\prime}}(d)<d$ it follows that in the Macaulay expension of $h_{I^{\prime}}(d)$ each $\epsilon_{i}$ is either 1 or 0 . Hence $h_{I^{\prime}}(d)^{\langle d\rangle}=h_{I^{\prime}}(d)$. From this it follows that $h_{I^{\prime}}(d+1) \leq h_{I^{\prime}}(d)$. If the inequality is strict then we are done, since then $h_{I}(d+1) \leq h_{I^{\prime}}(d+1)<h_{I}(d)$.

Suppose now that $h_{I^{\prime}}(d+1)=h_{I^{\prime}}(d)$ holds. Then Theorem 2.4 implies that the Hilbert function and Hilbert polynomial of $I^{\prime}$ coincide in every degree $\geq d$, and, moreover, that this polynomial is the constant polynomial $h_{I^{\prime}}(d)$. Hence $I_{d+1}^{\prime}$ has a base locus. Since $I_{d+1}^{\prime} \subset$ $I_{d+1}$ and $I_{d+1}$ is base point free it follows that

$$
h_{I}(d+1)<h_{I^{\prime}}(d+1) \leq h_{I}(d) .
$$

A final result of this type that we use is

Theorem 2.6 (Green, [11]) Let $V \subset \mathbf{C}\left[x_{0}, \ldots, x_{n}\right]_{d}$ be a linear system of codimension $c$. Let $H=\{\ell=0\}$ be a general hyperplane. Then the restriction of $V$ to $H$ has codimension at most $c_{\langle d\rangle}$ in $\left(\mathbf{C}\left[x_{0}, \ldots, x_{n}\right] / \ell\right)_{d}$. 


\section{Nodal complete intersections}

Notation 3.1 Let $n=2 k+1$ be a positive odd integer, $c$ be a positive integer, and $\left(w_{0}, \ldots, w_{n+c}\right)$ a sequence of positive integers. Let us denote with $\mathbf{P}:=\mathbf{P}\left(w_{0}, \ldots, w_{n+c}\right)$ the associated weighted projective space. Let $S=\mathbf{C}\left[x_{0}, \ldots, x_{n+c}\right]$ be the graded polynomial ring such that $\operatorname{deg} x_{i}=w_{i}$.

Definition 3.2 We say that a codimension $c$ complete intersection $X \subset \mathbf{P}$ is a nodal complete intersection of codimension $c$, if

(1) for all $p \in \mathbf{P}_{\text {sing }} \cap X$ we have that $X$ is quasi-smooth at $p$ and

(2) for all $p \in X_{\text {sing }} \backslash\left(\mathbf{P}_{\text {sing }} \cap X\right)$ we have that $(X, p)$ is a node.

Let $\Sigma$ denote the set $X_{\text {sing }} \backslash\left(\mathbf{P}_{\text {sing }} \cap X\right)$.

Proposition 3.3 Let $X \subset \mathbf{P}$ be a nodal complete intersection of codimension $c$ then for $i<n$

$$
\operatorname{dim} H^{i}(X)=\operatorname{dim} H^{i}(\mathbf{P}) .
$$

Moreover, for $i<n-1$ we have

$$
\operatorname{dim} H^{i}(X)=\operatorname{dim} H^{2 n-i}(X) .
$$

Proof The first equality follows from the Lefschetz hyperplane theorem [7, Theorem 4.2.6]. To prove the second equality we consider a partial resolution of singularities of $X$ :

Since $X$ is quasismooth outside $\Sigma$ we have that for all $i \neq 2 n$ and for all $p \in X \backslash \Sigma$ the group $H_{p}^{i}(X)$ vanishes. Let $\tilde{X}$ be the blow up of $X$ along $\Sigma$. Then $\tilde{X}$ is smooth along the exceptional divisor. In particular, for all $p \in \tilde{X}$ we have that $H_{p}^{i}(\tilde{X})=0$ if $i \neq 2 n$. This implies that $\tilde{X}$ is Q-homology manifold and satisfies Poincaré duality.

Consider the Mayer-Vietoris sequence associated with the discriminant square [19, Corollary-Definition 5.37]

$$
\cdots \rightarrow H^{i}(X) \rightarrow H^{i}(\tilde{X}) \oplus H^{i}(\Sigma) \rightarrow H^{i}(E) \rightarrow H^{i+1}(X) \rightarrow \ldots
$$

This is an exact sequence of mixed Hodge structures.

The exceptional divisor $E$ is the disjoint union of $\# \Sigma$ smooth quadrics in $\mathbf{P}^{n}$. Thus its cohomology can be nonzero only in even degree between 0 and $2 n-2$. Let $E_{j}$ and $E_{k}$ be distinct irreducible components of $E$. For $i$ even between 2 and $2 n-2 \operatorname{consider} c_{1}\left(E_{j}\right)^{i / 2} \in$ $H^{i}(\tilde{X})$. Then $c_{1}\left(E_{j}\right)^{i / 2}$ is mapped to zero in $H^{i}\left(E_{k}\right)$ and to a nonzero element of $H^{i}\left(E_{j}\right)$. If $i \neq n-1$ then $H^{i}\left(E_{j}\right)$ is one-dimensional and therefore the map $H^{i}(X) \rightarrow H^{i}(E)$ is surjective for even $i$, different from 0 and $n-1$. From this it follows that the above long exact sequence splits in the following exact sequences:

- $0 \rightarrow H^{0}(X) \rightarrow H^{0}(\tilde{X}) \oplus H^{0}(\Sigma) \rightarrow H^{0}(E) \rightarrow 0$;

- $0=H^{i}(X) \cong H^{i}(\tilde{X})$ for $i$ odd, different from $n$;

- $0 \rightarrow \mathbf{Q}=H^{i}(X) \rightarrow H^{i}(\tilde{X}) \rightarrow H^{i}(E) \rightarrow 0$ for even $i$ different from 0 and $n-1$;

- $0 \rightarrow H^{n-1}(X) \rightarrow H^{n-1}(\tilde{X}) \rightarrow H^{n-1}(E) \rightarrow H^{n}(X) \rightarrow H^{n}(\tilde{X})$.

Since $E$ is a disjoint union of smooth quadrics it follows that $h^{i}(E)=\# \Sigma=h^{2 n-i}(E)$ for $i \neq 0, n-1, n+1,2 n$. From Poincaré duality it follows that $h^{i}(\tilde{X})=h^{2 n-i}(\tilde{X})$ for all $i$. Combining this yields that that $h^{i}(X)=h^{2 n-i}(X)$ for $i \neq 0, n-1, n+1,2 n$.

To finish the proof, note that we showed that $h^{0}(X)=h^{0}(\tilde{X})=1$ and $h^{2 n}(X)=h^{2 n}(\tilde{X})=$ 1. 
The proof of the above result suggests that $h^{n+1}(X, \mathbf{Q})$ may be strictly larger than $h^{n-1}(X, \mathbf{Q})$.

Definition 3.4 The defect $\delta$ of $X$ equals $h^{n+1}(X, \mathbf{Q})-h^{n-1}(X, \mathbf{Q})$.

Remark 3.5 If $n=3$ then $\delta$ equals the rank of the group $\mathrm{CH}^{1}(X) / \operatorname{Pic}(X)$. Since this group is free, $\delta$ measures the failure of Weil divisors to be Cartier.

Lemma 3.6 Let $X$ be a nodal complete intersection. Let $\mathcal{D}$ be the equisingular deformation space of $X$. Then the locus

$$
\left\{X^{\prime} \in \mathcal{D} \mid \delta\left(X^{\prime}\right)=\delta(X)\right\}
$$

is a Zariski open subset of $\mathcal{D}$.

Proof Let $\left(X_{t}\right)_{t \in U}$ be an equisingular deformation of $X$. Possibly after shrinking $U$, we have that $X_{t}$ has the same number of nodes for all $t \in U$. Blowing up these nodes simultaneously yields a flat family $\tilde{X}_{t}$ of smooth projective varieties. Hence $H^{n+1}\left(\tilde{X}_{t}\right)$ is independent of $t$. Let $E$ be the exceptional divisor of the blow-up of a node, and let $s$ the number of nodes of $X$. As in the proof of Proposition 3.3 we can consider the Mayer-Vietoris sequence associated with the discriminant square. This time we take also into account the Hodge structures. We obtain the following exact sequence

$$
0 \rightarrow \mathrm{Gr}_{n+1}^{W} H^{n+1}\left(X_{t}\right) \rightarrow H^{n+1}\left(\tilde{X}_{t}\right) \rightarrow H^{n+1}(E)^{\oplus s} \rightarrow H^{n+2}\left(X_{t}\right) .
$$

Since $X_{t}$ is a nodal hypersurface and $n$ is odd we have that $H^{n+2}\left(X_{t}\right)=0$. Since all singularities of $X_{t}$ are nodes or induced by the ambient space it follows that $H^{n+1}\left(X_{t}\right)$ has a Hodge structure of pure weight $n+1$. This yields

$$
h^{n+1}\left(X_{t}\right)=h^{n+1}\left(\tilde{X}_{t}\right)-s \cdot h^{n+1}(E) \text {. }
$$

Both terms on the right hand side are independent of $t$, hence so is $h^{n+1}\left(X_{t}\right)$. By Proposition 3.3 we have $h^{n-1}\left(X_{t}\right)=1$ for all $t$ and hence $\delta\left(X_{t}\right)=h^{n+1}\left(X_{t}\right)-h^{n-1}\left(X_{t}\right)=$ $h^{n+1}\left(X_{t}\right)-1=h^{n+1}(X)-1=\delta(X)$.

One can express $\delta$ in terms of the Hilbert function of the ideal of the nodes. Suppose now that $c=1$, i.e., $X$ is a hypersurface. Set $m:=\frac{n+1}{2}$.

The following result is [6, Proposition 3.2]:

Proposition 3.7 Let $X \subset \mathbf{P}$ be a nodal hypersurface of degree d. Let $\Sigma \subset \mathbf{P}$ be the locus of the nodes of $X$. Then

$$
\delta(X)=\# \Sigma-\operatorname{dim}(S / I(\Sigma))_{m d-\sum w_{i}}
$$

\section{Hypersurfaces with positive defect}

We will use the results from the previous section to reprove the following result by Cheltsov on the minimal number of nodes to have positive defect:

Theorem 4.1 (Cheltsov, [2,3]) Let $X \subset \mathbf{P}^{4}$ be a nodal hypersurface of degree $d$, with $d \geq 3$. Assume that $h^{4}(X) \geq 2$, i.e., that $X$ has positive defect. Then $X$ has at least $(d-1)^{2}$ nodes. If $X$ has precisely $(d-1)^{2}$ nodes then $X$ contains a plane. 
Proof Without loss of generality we may assume that $X_{H}=X \cap V\left(x_{4}\right)$ is smooth. In particular, none of the nodes of $X$ is contained in $V\left(x_{4}\right)$. Set $R=\mathbf{C}\left[x_{0}, x_{1}, x_{2}, x_{3}, x_{4}\right]$ and $S=\mathbf{C}\left[x_{0}, x_{1}, x_{2}, x_{3}\right]$. Let $I \subset R$ be the ideal of the nodes of $X$. Then the Hilbert polynomial $p_{I}(t)$ is the constant polynomial $\# \Sigma$. Since $X$ has positive defect it follows from Proposition 3.7 that $h_{I}(2 d-5)<p_{I}(2 d-5)$.

Let $I_{H} \subset S$ be the ideal obtained by substituting $x_{4}=0$ in $I$. From the fact that none of the nodes of $X$ is contained in $V\left(x_{4}\right)$ it follows that the following sequence is exact:

$$
0 \rightarrow(R / I)_{k-1} \stackrel{x_{4}}{\rightarrow}(R / I)_{k} \rightarrow\left(S / I_{H}\right)_{k} \rightarrow 0 .
$$

If $h_{I_{H}}(2 d-4)$ vanishes then we have $h_{I_{H}}(k)=0$ for $k \geq 2 d-4$. In particular, $h_{I}(k)=$ $h_{I}(k+1)$ for $k \geq 2 d-5$. Since we know that $h_{I}(2 d-5)<p_{I}(2 d-5)$ this cannot be the case and hence $h_{I_{H}}(2 d-4)>0$ holds. Fix now a codimension one subspace $W$ of $S_{2 d-4}$ containing $\left(I_{H}\right)_{2 d-4}$. Define $I^{\prime} \subset S$ by $I_{e}^{\prime}=\left\{g \mid g S_{2 d-4-e} \subset W\right\}$ if $e \leq 2 d-4$ and $I_{e}^{\prime}=S_{e}$ for $e \geq 2 d-3$. Then $I^{\prime}$ is an ideal, containing $I_{H}$. By construction the pairing $\left(S / I^{\prime}\right)_{e} \times\left(S / I^{\prime}\right)_{2-d-e} \rightarrow\left(S / I^{\prime}\right)_{e}$ is perfect, hence $S / I^{\prime}$ is a Gorenstein ring with socle degree $2 d-4$. In particular, $h_{I^{\prime}}(k)=h_{I^{\prime}}(2 d-4-k)$.

Let $f$ be a defining polynomial for $X$. Since $\left(I_{H}\right)_{d-1}$ contains the partial derivative $\frac{\partial f}{\partial x_{i}}\left(x_{0}, x_{1}, x_{2}, x_{3}, 0\right)$ for $i=0, \ldots, 3$ and $X \cap V\left(x_{4}\right)$ is smooth, we have that $I_{d-1}^{\prime}$ is base point free.

If $h_{I^{\prime}}(k)<2 d-3-k$ for some $k$ with $d-2 \leq k \leq 2 d-4$, then from Corollary 2.5 it would follow that $h_{I^{\prime}}(2 d-4)=0$, contradicting the fact that $h_{I^{\prime}}(2 d-4)=1$. Hence $h_{I^{\prime}}(k) \geq 2 d-3-k$ for every integer $k$ such that $d-2 \leq k \leq 2 d-4$. Combining this information we obtain

$$
p_{I}=p_{I}(2 d-4) \geq h_{I}(2 d-4)=\sum_{i=0}^{2 d-4} h_{I_{H}}(i) \geq \sum_{i=0}^{2 d-4} h_{I^{\prime}}(i) \geq(d-1)^{2} .
$$

If $p_{I}=(d-1)^{2}$ then we have that $h_{I}$ equals the Hilbert function of a complete intersection of degree $(1,1, d-1, d-1)$. Since $I$ contains the partials of $f$ it follows that the linear system $\left|I_{d-1}\right|$ has finitely many base points.

Now $I$ has two generators in degree 1 and two further generators in degree $d-1$. In particular these four generators define a codimension four scheme and hence these four generators form a regular sequence. The Hilbert function of the ideal generated by these four forms equals the Hilbert function of $I$. Hence $I$ is a complete intersection ideal, generated by $f_{1}, f_{2}, f_{3}, f_{4}$, with $\operatorname{deg}\left(f_{1}\right)=\operatorname{deg}\left(f_{2}\right)=1, \operatorname{deg}\left(f_{3}\right)=\operatorname{deg}\left(f_{4}\right)=d-1$.

At each node of $X$ the polynomials $f_{1}, f_{2}, f_{3}, f_{4}$ induce a local system of coordinates. Since at each singular point of $X$ the polynomial $f$ vanishes up to order two it follows that $f$ is an element of the ideal generated by the $f_{i} f_{j}$ with $i \leq j$. These forms have degree at most $d$ if and only if $i \leq 2$. In particular, $f$ is in the ideal generated by $f_{1}$ and $f_{2}$ and therefore contains the plane $f_{1}=f_{2}=0$.

Remark 4.2 The proof reveals also the following interesting observation. Suppose $I$ is the ideal of the nodes of a threefold of degree $d$ with positive defect. Then

$$
h_{I}(d) \geq \sum_{k=0}^{d} h_{I^{\prime}}(k) \geq \frac{1}{2}\left(d^{2}+3 d-10\right)
$$

Recall that $I_{d}$ is the tangent space to the equisingular deformation space of $X$ [13]. Hence it follows that any family of degree $d$ nodal hypersurfaces with positive defect has codimension 
at least $\frac{1}{2}\left(d^{2}+3 d-10\right)$ in $S_{d}$. Moreover, if equality holds then the above proof shows that $X$ contains a plane.

Consider now hypersurfaces containing a fixed plane $P$. They form a family of codimension $\frac{1}{2}(d+1)(d+2)$. Since the Grassmannian of planes in $\mathbf{P}^{4}$ has codimension 6 it follows that the total family has codimension $\frac{1}{2}\left(d^{2}+3 d-10\right)$. A general element of this family is of the form $\ell_{1} f_{1}+\ell_{2} f_{2}$ with $\operatorname{deg}\left(\ell_{i}\right)=1$ and $\operatorname{deg}\left(f_{i}\right)=d-1$. In particular, a general element is a nodal hypersurface. Hence the largest-dimensional family of nodal hypersurfaces with positive defect consists of hypersurfaces containing a plane.

The bound we obtained for $h_{I_{H}}(k)$ (for $d-1 \leq k \leq 2 d-4$ ) is also used in some of the proofs for the explicit Noether-Lefschetz theorem for surfaces in $\mathbf{P}^{3}$ (e.g., see [12]). However, if $n>3$ then Corollary 2.5 is insufficient to deduce the explicit Noether-Lefschetz theorem. Similarly, we were not able to deduce a good lower bound for the number of nodes to have positive defect from this Corollary. To obtain an explicit Noether-Lefschetz theorem in higher (even) dimension Otwinowska [17] proved a result on the Hilbert function of ideals containing the ideal of a certain complete intersection. This result seems still to be insufficient to obtain a sharp lower bound for the number of nodes to have positive defect. However, Otwinowska's result is strong enough to determine the largest component of the locus of nodal hypersurfaces with positive defect. Moreover, if the famous conjecture [8, Conjecture $V_{m}$ ] of Eisenbud, Green and Harris on the Hilbert function of ideals containing a complete intersection holds true, then the result of Otwinowska is strong enough to deduce the minimal number of nodes.

Notation 4.3 Let us define $p_{n, d}=\left(\begin{array}{c}d+n+1 \\ n+1\end{array}\right)-(n+1)(n+2)$. Then $p_{n, d}$ equals the Hilbert function of a complete intersection of multidegree $\left(1^{n+1},(d-1)^{n+1}\right)$ evaluated in degree $d$, if $d>2$.

Consider a hypersurface $X \subset \mathbf{P}^{2 n+2}$ of the form $\sum_{i=0}^{n} x_{i} f_{i}$, with $\operatorname{deg}\left(f_{i}\right)=d-1$. If the $f_{i}$ are chosen sufficiently general then the singular locus is $x_{0}=\cdots=x_{n}=f_{0}=\cdots=$ $f_{n}=0$. This is a complete intersection of multidegree $\left(1^{n+1},(d-1)^{n+1}\right)$. The tangent space to the equisingular deformation space has codimension $p_{n, d}$ and an easy calculation shows that this space is reduced, i.e., the actual deformation space has the same codimension.

Theorem 4.4 Fix $n \in \mathbf{N}, n \geq 2$. Let $\mathrm{DEF}_{d} \subset \mathbf{C}\left[x_{0}, \ldots, x_{2 n+2}\right]_{d}$ be the locus of nodal hypersurfaces with positive defect. Then there exists a $D$ such that if $d>D$ and $L$ is an irreducible component of $\mathrm{DEF}_{d}$ then codim $L \geq p_{n, d}$.

Moreover, if Conjecture 1 of [17] holds then we may take $D=2$ and any hypersurface in $\mathrm{DEF}_{d}$ has at least $(d-1)^{n+1}$ nodes.

Proof Let $X \in L$. From Lemma 3.6 it follows that a general equisingular deformation of $X$ also has positive defect, i.e., $L$ is also an irreducible component of the equisingular deformation space of $X$.

Fix a general hyperplane $H$. Since $X$ has positive defect there is a class $\gamma$ in $H_{2 n+2}(X, \mathbf{Q})$ which is not the multiple of the intersection of classes of hyperplanes. The intersection product of $\gamma$ with $H$ yields a nonzero Hodge class in $H^{2 n}\left(X_{H}, \mathbf{Q}\right)_{\text {prim. }}$. The Noether-Lefschetz locus of hypersurfaces of degree $d$ in $\mathbf{P}^{2 n+1}$ parametrizes hypersurfaces having a nonzero Hodge

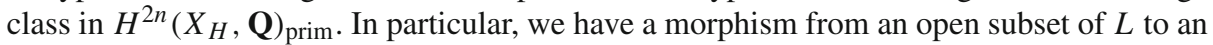
irreducible component $\mathrm{NL}\left(\gamma_{H}\right)$ of this Noether-Lefschetz locus. The differential of this map defines a map $d_{H}$ from the tangent space $T_{X} L$ to the tangent space of $\mathrm{NL}\left(\gamma_{H}\right)$ at $X_{H}$.

Let $F$ be a defining polynomial for $X$. The tangent space $T_{X} L$ can be identified with the degree $d$ part of the saturation of the Jacobian ideal of $F$. Without loss of generality we may 
assume that $H=\left\{x_{2 n+2}=0\right\}$. We have that $\left.J_{d}(F)\right|_{x_{2 n+2}=0}$ is contained in $T_{X_{H}} \operatorname{NL}\left(\gamma_{H}\right)$. As explained in [17] there exists an ideal $I \subset \mathbf{C}\left[x_{0}, \ldots, x_{2 n+1}\right]$, such that $T_{X} \mathrm{NL}\left(\gamma_{H}\right)$ is contained in $I_{d}$ and $\mathbf{C}\left[x_{0}, \ldots, x_{2 n+1}\right] / I$ is an Artinian Gorenstein ring with socle degree $(n+1) d-2 n-2$. Since $X_{H}$ is smooth we have that $I$ contains a complete intersection of multidegree $(d-1)^{2 n+2}$. Hence we can apply [17, Théorème 1]. From this it follows that there is a constant $D$ depending on $n$ such that for $d \geq D$ we have codim $I_{d} \geq\left(\begin{array}{c}d+n \\ n\end{array}\right)-(n+1)^{2}$.

Let $J$ be the ideal of the nodes of $X$. Then

$$
\left.J\left(F\left(x_{0}, \ldots, x_{2 n+1}, 0\right)\right) \subset J\right|_{x_{2 n+2}=0} \subset I .
$$

If codim $I_{d}=\left(\begin{array}{c}d+n \\ n\end{array}\right)-(n+1)^{2}$ holds then we have by [17, Théorème 1] that $I$ up to degree $d$ coincides with a complete intersection ideal of multidegree $\left(1^{n+1},(d-1)^{n+1}\right)$. In this case $h_{J}(d)$ is at least

$$
\sum_{k=0}^{d} h_{I}(k)=\sum_{k=0}^{d}\left(\begin{array}{c}
k+n \\
n
\end{array}\right)-(n+1)-(n+1)^{2}=p_{n, d} .
$$

If the codimension of $I_{d}$ is larger than $\left(\begin{array}{c}d+n \\ n\end{array}\right)-(n+1)^{2}$ then $\mathrm{NL}\left(\gamma_{H}\right)$ is different from the component of NL parametrizing hypersurfaces containing an $n$-dimensional linear space. From [18] it follows that for $d$ sufficiently large, the largest component of this type consists of hypersurfaces containing a quadric of dimension $n$. This locus has codimension

$$
c_{0}:=\left(\begin{array}{c}
d+n+1 \\
n+1
\end{array}\right)-\left(\begin{array}{c}
d+n-1 \\
n+1
\end{array}\right)-\frac{3 n^{2}+9 n+4}{2} .
$$

If $n \geq 16$ then the Macaulay expansion of $c_{0}$ equals

$$
\left(\begin{array}{c}
d+n \\
d
\end{array}\right)+\sum_{i=4}^{d-1}\left(\begin{array}{c}
i+n-1 \\
i
\end{array}\right)+\left(\begin{array}{c}
n-1 \\
3
\end{array}\right)+\left(\begin{array}{c}
n-5 \\
2
\end{array}\right)+\left(\begin{array}{c}
n-15 \\
1
\end{array}\right) .
$$

For $n \leq 15$ we have that the the Macaulay expansion of $c_{0}$ equals

$$
\left(\begin{array}{c}
d+n \\
d
\end{array}\right)+\sum_{i=6}^{d-1}\left(\begin{array}{c}
i+n-1 \\
i
\end{array}\right)+\sum_{i=1}^{5}\left(\begin{array}{c}
i+a_{i} \\
i
\end{array}\right)
$$

with $n-1 \geq a_{7} \geq a_{6} \geq \cdots \geq a_{1} \geq-1$.

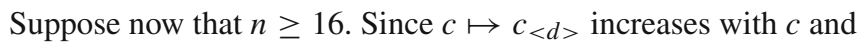

$$
h_{J}(d)_{<d>} \geq h_{J_{H}}(d) \geq c_{0}
$$

(Theorem 2.6) we have that $h_{J}(d)$ is at least

$$
\left(\begin{array}{c}
d+n+1 \\
d
\end{array}\right)+\sum_{i=4}^{d-1}\left(\begin{array}{c}
i+n \\
i
\end{array}\right)+\left(\begin{array}{c}
3+n-3 \\
3
\end{array}\right)+\left(\begin{array}{c}
2+n-6 \\
2
\end{array}\right)+\left(\begin{array}{c}
1+n-15 \\
1
\end{array}\right) .
$$

In particular, there exists a constant $C_{n}$ depending only on $n$ such that the right hand side equals $\left(\begin{array}{c}d+n+1 \\ d\end{array}\right)+\left(\begin{array}{c}d+n \\ d\end{array}\right)-C_{n}$. Therefore we have that for $d$ sufficiently large $h_{J}(d)>p_{n, d}$ holds. If $n<16$ then a similar argument will yield the proof for large $d$.

Suppose now that [17, Conjecture 1] holds. Let $I^{\prime} \subset S_{H}$ be the ideal of a complete intersection of multidegree $\left(1^{n+1},(d-1)^{n+1}\right)$. Then [17, Conjecture 1] implies $h_{I}(k) \geq$ 
$h_{I^{\prime}}(k)$ for all $k \leq(n+1) d-2 n-1$. In particular,

$$
p_{J} \geq \sum_{k=0}^{n d-2 n-2} p_{J_{H}}(k) \geq \sum_{k=0}^{n d-2 n-2} h_{I^{\prime}}(k)=(d-1)^{n} .
$$

and

$$
h_{J}(d) \geq \sum_{k=0}^{d} p_{J_{H}}(k) \geq \sum_{k=0}^{d} h_{I^{\prime}}(k)=p_{n, d} .
$$

Remark 4.5 Otwinowska shows in [17] that [17, Conjecture 1] is implied by the EisenbudGreen-Harris conjecture on the Hilbert function of ideals containing a complete intersection.

We switch now to the case of double covers.

Theorem 4.6 Let $f \in \mathbf{C}\left[x_{0}, x_{1}, x_{2}, x_{3}\right]$ be a squarefree polynomial of degree $2 d$, such that $V(f)$ is a nodal surface. Let $X: y^{2}=f$ be the double cover branched along $f$. Suppose $h^{4}(X)>1$. Then $X$ has at least $d(2 d-1)$ nodes. If $d \geq 2$ holds and $X$ has precisely $d(2 d-1)$ nodes then there exist forms $\ell, g, h$ of degree $1, d$ and $2 d-1$ respectively such that $f=\ell g+h^{2}$.

Proof Without loss of generality we may assume that $X_{H}=X \cap V\left(x_{3}\right)$ is smooth, in particular, none of the nodes of $X$ is contained in $V\left(x_{3}\right)$. Let $R=\mathbf{C}\left[x_{0}, x_{1}, x_{2}, x_{3}\right]$ and $S=\mathbf{C}\left[x_{0}, x_{1}, x_{2}\right]$. Let $I \subset R$ be the ideal of the nodes of $V(f)$. Note that the nodes of $X$ correspond one-to-one with the nodes of $V(f)$. Moreover, since $y$ is in the Jacobian ideal of $X$ we have that the Jacobian rings of $X$ and of $V(f)$ are isomorphic.

Since $h^{4}(X) \geq 2$ it follows from Proposition 3.7 that we have $h_{I}(3 d-4)<p_{I}(3 d-4)$. Let $I_{H} \subset S$ be the ideal obtained by substituting $x_{3}=0$ in $I$. Consider the exact sequence

$$
0 \rightarrow(R / I)_{k-1} \stackrel{x_{3}}{\rightarrow}(R / I)_{k} \rightarrow\left(S / I_{H}\right)_{k} \rightarrow 0 .
$$

As in the proof of Theorem 4.1 we obtain that $h_{I_{H}}(3 d-3)>0$. Fix a codimension one subspace $W$ of $S_{3 d-3}$ containing $\left(I_{H}\right)_{3 d-3}$. Define $I^{\prime} \subset S$ by $I_{e}^{\prime}=\left\{f \mid f S_{3 d-3-e} \subset W\right\}$ if $e \leq 3 d-3$ and $I_{e}^{\prime}=S_{e}$ for $e \geq 3 d-2$. Then $I^{\prime}$ is an ideal, containing $I_{H}$. Moreover $S / I^{\prime}$ is a Gorenstein ideal with socle degree $3 d-3$ and hence $h_{I^{\prime}}(k)=h_{I^{\prime}}(3 d-3-k)$.

The linear system $I_{2 d-1}^{\prime}$ contains the partials of $f$ specialized at $x_{3}=0$ and since $X_{H}$ is smooth this linear system must be base point free. From Corollary 2.5 we obtain that $h_{I^{\prime}}(k) \geq 3 d-2-k$ for $2 d-2 \leq k \leq 3 d-2$. Using Gorenstein duality it follows that $h_{I^{\prime}}(k) \geq k+1$ for $k \leq d-1$. Theorem 2.1 implies that $h_{I^{\prime}}(k) \geq d$ for $d \leq k \leq 2 d-2$. Combining everything we obtain

$$
\begin{aligned}
p_{I} & =p_{I}(3 d-3) \geq h_{I}(3 d-3)=\sum_{i=0}^{3 d-3} h_{I_{H}}(i) \\
& \geq 2 \sum_{i=0}^{d-1}(i+1)+d(d-2)=d(d+1)+d(d-2)=d(2 d-1) .
\end{aligned}
$$

Suppose now that $p_{I}$ is exactly $d(2 d-1)$. Then we have $h_{I}(1)=3$. In particular there is a linear form $\ell$ that vanishes at all the nodes. If $\ell$ is a factor of $f$ then we can write $f=\ell f_{1}$. All the nodes of $V(f)$ are contained in $V\left(\ell, f_{1}\right)$ which consists of $2 d-1$ points. Since we 
know that $X$ has at least $d(2 d-1)$ nodes this cannot happen and therefore $\ell$ is not a factor of $f$.

Assume that $\ell=x_{3}$ and write $f=f_{0}\left(x_{0}, x_{1}, x_{2}\right)+x_{3} g\left(x_{0}, x_{1}, x_{2}, x_{3}\right)$. If $p$ is a node of $V(f)$ then $g$ vanishes at $p$ and $p$ is a double point of $f_{0}=0$. If $f_{0}$ contains a component with multiplicity at least three then $X$ contains a singularity which is not a node, in particular, we can write $f_{0}=f_{1}^{2} f_{2}$, such that $f_{1}$ and $f_{2}$ are coprime and both are squarefree. Hence the locus of the nodes of $V(f)$ consists of points $p$ such that $f_{1}\left(x_{0}, x_{1}, x_{2}\right)=g\left(x_{0}, x_{1}, x_{2}, 0\right)=0$ together with points $p$ such that $g\left(x_{0}, x_{1}, x_{2}, 0\right)=0$ and $p$ is a double point of $f_{2}$.

Denote with $e_{i}$ the degree of $f_{i}$. Then there are precisely $e_{1}(2 d-1)$ points of the former type and at most $\frac{1}{2}\left(e_{2}-1\right) e_{2}$ points of the second type. Their sum is strictly less than $d(2 d-1)$ if $e_{1} \neq 0,2 d$. If $e_{1}$ were $2 d$ then the set of nodes of $V(f)$ is also the set of nodes of a (reducible) plane curve of degree $2 d$. From [15, Proposition 3.6] it follows that then $h_{I}(k)=d(2 d-1)$ holds for $k \geq 2 d-2$, contradicting $h_{I}(3 d-3)<d(2 d-1)$. Hence $e_{1}=d$ and $f_{0}$ is a square.

Example 4.7 In order to show that the bound $d(2 d-1)$ for the number of nodes is sharp, consider $y^{2}=h^{2}+\ell g$ with $\operatorname{deg}(f)=d$ and $\operatorname{deg}(g)=2 d-1$. Then for general $f, g, \ell$ the singular locus is a complete intersection of multidegree $(1, d, 2 d-1)$, i.e., it consists of $d(2 d-1)$ points. Moreover $\ell=y-f=0$ defines a Weil divisor that is not $\mathbf{Q}$-Cartier and hence the double cover has positive defect.

\section{The Ciliberto-Di Gennaro conjecture}

In this section we prove the following conjecture for $d \geq 7$. This conjecture is trivially true for $d=1,2$ and for $d=3$ this conjecture follows from Theorem 4.1. As mentioned in the Introduction, this conjecture has been proven for $d=3$ by [9] and for $d=4$ by [4,14,20]. Hence this conjecture remains open for $d=5,6$. We start by recalling the conjecture.

Conjecture 5.1 Let $X \subset \mathbf{P}^{4}$ be a non-factorial nodal threefold of degree $d$ with at most $2(d-2)(d-1)$ nodes then either $X$ contains a plane or a quadric surface and if $X$ contains a quadric surface then $X$ has precisely $2(d-2)(d-1)$ nodes.

Note that in [5] Ciliberto-Di Gennaro proved a weaker form of this conjecture, namely they showed that if $X$ is non-factorial and has at most $2(d-2)(d-1)$ nodes then $X$ contains a plane, a quadric surface or a singular surface.

Lemma 5.2 Let $I \subset S:=\mathbf{C}\left[x_{0}, \ldots, x_{n}\right]$ be a homogeneous ideal such that $S / I$ is Artinian Gorenstein of socle degree $N$. Let $d_{k}$ be the smallest integer $t$ such that the dimension of the base locus of $I_{t}$ is at most $k$. Then

$$
\sum_{k=-1}^{n-1} d_{k} \geq N+n+1
$$

Proof The ideal $I$ contains a complete intersection ideal $I^{\prime}$ of multidegree $\left(d_{n-1}, \ldots, d_{-1}\right)$. In particular, $I_{k}^{\prime}=\mathbf{C}\left[x_{0}, \ldots, x_{n}\right]_{k}$ for $k>\sum_{i=-1}^{n-1}\left(d_{i}-1\right)$. Since $I_{k}^{\prime}$ is contained in $I_{k}$ and $I_{N} \neq \mathbf{C}\left[x_{0}, \ldots, x_{n}\right]_{N}$ we have $\sum_{i=-1}^{n-1}\left(d_{i}-1\right) \geq N$.

Lemma 5.3 Suppose $d \geq 6$. Let $X \subset \mathbf{P}^{4}$ be a nodal threefold of degree $d$ with at most $2(d-2)(d-1)$ nodes. Assume that $X$ has positive defect. Let $J$ be the ideal of $X_{\text {sing, }}$, 
$H=\{\ell=0\}$ a general hyperplane, $J_{H}=(J, \ell)$. Let I be an ideal containing $J_{H}$, such that $S / I$ is Artinian Gorenstein of socle degree $2 d-4$.

If $h_{I}(d-4) \leq 2 d-7$ then $X_{\text {sing }}$ contains a subset which is a complete intersection of multidegree $(1,1, d-1, d-1)$ or of multidegree $(1,2, d-2, d-1)$.

Proof Let $\Sigma \subset X_{\text {sing }}$ be a minimal subset such that the linear system of polynomials of degree $2 d-5$ vanishing at $\Sigma$ has defect one. Let $J=I(\Sigma)$. Without loss of generality we may assume $X \cap V\left(x_{4}\right)$ is smooth. In particular we have that $\Sigma \subset V\left(x_{4}\right)=\emptyset$. Let $J_{H}$ be the ideal obtained by substituting $x_{4}=0$ in $H$. Then $J_{H}$ is an ideal of $S:=\mathbf{C}\left[x_{0}, \ldots, x_{3}\right]$. As in the proof of Theorem 4.1 we obtain that $h_{J_{H}}(2 d-4)=1$ and $h_{J_{H}}(2 d-5)=0$. Let $I$ be the ideal containing $J_{H}$, such that $S / I$ is Artinian Gorenstein of socle degree $2 d-4$.

Suppose that $h_{I}(d-4) \leq 2 d-7$. Then from the proof of [21, Proposition 1.1] in Section 1 of loc. cit. it follows that there exists either a line $L$ or a conic $C$ such that $I_{k}=I(L)_{k}$ for $k \leq d-4$ or $I_{k}=I(C)_{k}$ for $k \leq d-4$.

Suppose first that $I_{d-4}$ is the degree $d-4$-part of the ideal of a line $L$. Using the notation of Lemma 5.2 we have that $d_{3}=d_{2}=d_{1}=1$. Since the base locus of $I_{d-1}$ is empty it follows that $d_{0} \leq d_{-1} \leq d-1$. From Lemma 5.2 it follows that $\sum_{i=-1}^{3} d_{i} \geq 2 d+1$. In particular, $d_{0}=d_{-1}=d-1$ holds and $I$ contains a complete intersection ideal $I^{\prime}$ of multidegree $(1,1,1, d-1, d-1)$. Since both $S / I$ and $S / I^{\prime}$ are Artinian Gorenstein rings of socle degree $2 d-4$ we have $I=I^{\prime}$.

We are now going to show that the base locus $B$ of $J_{d-2}$ contains a plane. We claim that every component of $B$ has dimension at most 2 , and that $B$ contains a component of dimension 2:

Suppose that first the base locus of $J_{d-2}$ would have dimension at least 3 . Then $h_{J}(d-2) \geq$ $h_{\mathbf{P}^{3}}(d-2)=\frac{1}{6}(d+1) d(d-1)$. This would imply that

$$
\begin{aligned}
h_{J}(2 d-4) & =h_{J}(d-2)+\sum_{k=d-1}^{2 d-4} h_{J_{H}}(k) \\
& \geq \frac{1}{6}(d+1) d(d-1)+\frac{1}{2}(d-1)(d-2) \\
& >2(d-1)(d-2)
\end{aligned}
$$

contradicting the fact that the length of $V(J)$ is at most $2(d-2)(d-1)$.

Hence the base locus $B$ of $J_{d-2}$ is of dimension at most two. The base locus of $I_{d-2}$ is a line $L$ and is contained in $B \cap H$. Hence one of the irreducible components of $B$ is a plane $P$.

We will now show that $P \subset X$. For this it suffices to show that $L \subset X_{H}$. Recall that $J_{d}$ is the tangent space to space of deformations of $X$ where the points in $\Sigma$ deform to nodes. These equisingular deformations have then also positive defect. Hence $I$ is contained in the tangent space $T \mathrm{NL}(\gamma)$ of the component $\mathrm{NL}(\gamma)$ of the Noether-Lefschetz locus of smooth surfaces of degree $d$ in $H$. From standard arguments in Noether-Lefschetz theory it follows that $T \mathrm{NL}(\gamma) \otimes S_{d-4}$ has codimension at least one in $S_{2 d-4}$ (see e.g., [12]). This space contains $I_{d} \otimes S_{d-4}$ and since $I$ is generated in degree $<d$, this latter space equals $I_{2 d-4}$. Since $I_{2 d-4}$ has codimension one, it follows $T$ NL $(\gamma) \otimes S_{d-4}=I_{2 d-4}$.

As remarked above, $I_{d} \subset T \mathrm{NL}(\gamma)$. From the results of [12] it follows that $\operatorname{codim} T \mathrm{NL}(\gamma) \geq d-3$. Since $I \subset \mathbf{C}\left[x_{0}, x_{1}, x_{2}, x_{3}, x_{4}\right]$ is a complete intersection ideal of multidegree $(1,1,1, d-1, d-1)$ it follows that $h_{I}(d)=d-3$, hence $T \operatorname{NL}(\gamma)=I_{d}$. In particular $X_{H}$ and $T \mathrm{NL}(\gamma)$ are as in [12, page 299-301]. From the argument presented there it follows that the line $L$ is contained in $X_{H}$. 
Suppose we are now in the case that $I_{d-4}$ is the degree $d-4$ part of the ideal of a conic. Without loss of generality we may assume that the conic is defined by $x_{0}=x_{1}=$ $f\left(x_{2}, x_{3}, x_{4}\right)=0$. Since $I_{d-1}$ is base point free we can find two further elements $f_{1}, f_{2} \in I$ of degree at most $d-1$, such that $x_{0}, x_{1}, f, f_{1}, f_{2}$ form a regular sequence of multidegree $\left(d_{3}, d_{1}, d_{1}, d_{0}, d_{-1}\right)$. From Lemma 5.2 it follows that $d_{0}+d_{-1} \geq 2 d-3$. Since $d_{-1} \leq d-1$ we have two possibilities, namely $\left(d_{0}, d_{-1}\right)$ equals $(d-2, d-1)$ or $(d-1, d-1)$.

Suppose first that $d_{0}=d-2$. Then $I$ contains a complete intersection ideal $I^{\prime}$ of $(1,1,2, d-2, d-1)$. From $I_{2 d-4}=I_{2 d-4}^{\prime}$ it follows that $I=I^{\prime}$. From this it follows that $J$ is contained in a complete intersection ideal of multidegree $(1,2, d-2, d-1)$. Using that $p_{J} \leq 2(d-2)(d-1)$ and that $p_{I^{\prime}}=2(d-2)(d-1)$ it follows that $J=I^{\prime}$ and that $X_{\text {sing }}$ is a complete intersection of multidegree $(1,2, d-2, d-1)$.

Suppose now that $d_{0}=d-1$. Then $I$ contains a complete intersection ideal $I^{\prime}$ of multidegree $(1,2, d-1, d-1)$. From e.g. [17, Sect. 1] it follows that if $I_{1}$ and $I_{2}$ are homogeneous ideals such that $I_{1} \subset I_{2}$ and both $S / I_{1}$ and $S / I_{2}$ are Artinian Gorenstein of socle degree $N+k$ and $N$ then $I_{2}=\left(I_{1}: F\right)$ for some form $F$ of degree $k$. We can apply this to $\left(I_{1}, I_{2}\right)=\left(I^{\prime}, I\right)$ and we find that $I=\left(I^{\prime}: h\right)$ for some linear form $h$. Note that the base locus of $I_{d-2}^{\prime}$ consists of a conic. If the base locus of $I_{d-2}$ is also a conic then we have that $h_{I}(k)=2 k+1$ for $k \leq d-2$. Using Gorenstein duality we get that

$$
\sum_{k=0}^{2 d-4} h_{I}(k) \geq 2 \sum_{k=0}^{d-3} 2 k+1+2 d-3=2(d-2)(d-1)+1
$$

Since $J_{H} \subset I$ it follows that $p_{J} \geq h_{J}(2 d-4)>2(d-2)(d-1)$, a contradiction. Hence the base locus of $I_{d-2}$ is not a conic. Since the base locus of $I_{d-4}$ is a conic and the base locus of $I_{d-2}$ is one dimensional we have that the conic is a union of two line lines $h_{1} h_{2}=0$ and one of the lines, say $h_{1}=0$ is contained in the base locus of $I_{d-2}$ and the linear form $h=h_{2}$. Recall that $I=\left(I^{\prime}: h_{2}\right)$. Since $h_{1} h_{2} \in I^{\prime}$ it follows that $h_{1} \in I$ and therefore that the base locus of $I_{1}$ is contained in a line, a contradiction, hence $d_{0}=d-1$ is impossible.

Lemma 5.4 Supposed $\geq 7$. Let $X \subset \mathbf{P}^{4}$ be a nodal threefold of degree $d$ with positive defect. Let $J$ be the Jacobian ideal of $X, H=\{\ell=0\}$ a general hyperplane, $J_{H}=(J, \ell)$. Let $I$ be an ideal containing $J_{H}$, such that $I$ is Artinian Gorenstein of socle degree $2 d-4$.

If $h_{I}(d-4)>2 d-7$ then $X_{\text {sing }}$ consists of at least $2(d-1)(d-2)+1$ points.

Proof Let $h(k)=2 k+1$ for $k \leq d-3, h(d-2)=2(d-2)$ and $h(k)=h(2 d-4-k)$ for $d-1 \leq k \leq 2 d-4$. Then $h$ is the Hilbert function of a complete intersection ideal of multidegree $(1,2, d-2, d-1)$.

Suppose that for some $k$ we have that $h_{I}(k)>2 k+1$. Then from Theorem 2.1 it follows that $h_{I}(j)>2 j+1=h(j)$ for $2 \leq j \leq k$.

In our case we have that $h_{I}(d-4)>2 d-7$. Hence $h_{I}(k)>h(k)$ for $2 \leq k \leq d-4$. Using Gorenstein duality we get $h_{I}(k)>h(k)$ for $d \leq k \leq 2 d-6$. In particular,

$$
\sum_{k \neq d-3, d-2, d-1} h_{I}(k)-h(k) \geq 2(d-5)=2 d-10 .
$$

From $h_{I}(d) \geq 2 d-6$ it follows from Theorem 2.1 that $h_{I}(d-1) \geq 2 d-7$. However, if $h_{I}(d-1)$ equals $2 d-7$ then $h_{I}(d)$ equals $2 d-6$ and the Hilbert polynomial of the base locus of $I_{d-1}$ equals $k+(d-6)$ by Theorem 2.4. In particular, the base locus of $I_{d}$ contains a line, which contradicts the fact that it is empty. Hence $h_{I}(d-1) \geq 2 d-6$. Similarly, if $h_{I}(d-2)=2 d-7$ then $I_{d-1}$ has a base component. Since $I_{d-1}$ is base point free this is 
not possible. This implies that $\sum_{k=d-3}^{d-1} h_{I}(k)-h(k) \geq-4$. In particular, if $2 d-14>0$ then $\sum h_{I}(k)>\sum h(k)=2(d-2)(d-1)$. This finishes the proof in the case $d>7$.

If $d=7$ then the above argument yields $\sum h_{I}(k) \geq 2(d-2)(d-1)$. We have to exclude equality. Suppose now that equality holds. Then all the inequalities in the previous paragraph become equalities. In particular,

$$
\begin{gathered}
h_{I}(d)=h_{I}(d-4)=2 d-6, h_{i}(d-3)=h_{i}(d-1)=2 d-6, \\
h_{I}(d-2)=2 d-6 \text { and } h_{i}(k)=h(k)+1 \text { for } k \in\{2,3,8,9\}
\end{gathered}
$$

Hence $h_{I}$ takes the following values $1,3,6,8,8,8,8,8,6,3,1$. This implies that the base locus in degree 1 and 2 is a plane. The base locus of $I_{3}$ has dimension at most one. From Lemma 5.2 it follows that the dimension of the base locus is at least one. Since $h_{I}(3)=8$ it follows that the base locus of $I_{3}$ is the intersection of two plane cubics having either a line or a conic as a common component.

From Lemma 5.2 it follows that $I$ contains a complete intersection ideal $I^{\prime}$ of multidegree $(1,3,4,6),(1,3,5,5),(1,3,5,6)$ or $(1,3,6,6)$.

In the first two cases we would have that the socle degree of $S / I^{\prime}$ is 10 . Since $S / I$ has also socle degree 10 this implies that $I=I^{\prime}$. However $I$ and $I^{\prime}$ have different Hilbert functions, hence this is not the case.

If $I^{\prime}$ is of multidegree $(1,3,5,6)$ then there exists a linear form $h$ such that $I=\left(I^{\prime}: h\right)$. From this it follows that the base locus of $I_{k}^{\prime}$ is contained in the base locus of $I_{k-1}^{\prime}$ union with $V(h)$. Note that the base locus of $I_{4}^{\prime}$ is a cubic curve $C$ and the base locus of $I_{3}$ is the intersection of two cubics. Hence the base locus of $I_{3}$ is a conic $Q=0$ together with a point and $V(h)$ is a component of $C$. Moreover we have that $Q h \in I_{3}^{\prime}$. This implies that $Q \in\left(I^{\prime}: h\right)=I$, contradicting that $h_{I}(2)=6$, hence we can exclude this case.

If $I^{\prime}$ is of multidegree $(1,3,6,6)$ then there exists a quadratic form $h$ such that $I=\left(I^{\prime}: h\right)$. From this it follows that the base locus of $I_{k}^{\prime}$ is contained in the base locus of $I_{k-2}^{\prime}$ union with $V(h)$. Note that the base locus of $I_{5}^{\prime}$ is a cubic curve $C$ and the base locus of $I_{3}$ is the intersection of two cubics. Hence the base locus of $I_{3}$ is contains either a line $L=0$ or a conic $Q=0$, and this curve is a component of $C$. In the first case we have $L h \in I_{3}^{\prime}$ and by construction that $L \in I_{1}$, a contradiction. In the second case we have that $h=h_{1} h_{2}$ and $Q h_{1} \in I_{3}^{\prime}$. This implies that $Q h_{1} h_{2} \in I_{4}^{\prime}$ and that $Q \in\left(I^{\prime}: h\right)=I$. A contradiction.

Theorem 5.5 (Ciliberto-Di Gennaro conjecture) Suppose $d \geq 7$. Let $X$ be a nodal hypersurface of degree $d$, with at most $2(d-1)(d-2)$ nodes. Then one of the following holds

(1) $X$ is factorial.

(2) X contains a plane and $X$ has at least $(d-1)^{2}$ nodes.

(3) X contains a quadric surface and $X$ has at least $2(d-1)(d-2)$ nodes.

Proof Suppose $X$ is not factorial. It follows directly from Lemma 5.3 and 5.4 that $X_{\text {sing }}$ contains a complete intersection $\Sigma$ either of multidegree $(1,1, d-1, d-1)$ or of multidegree $(1,2, d-2, d-1)$. In the first case $\Sigma$ consists of $(d-1)^{2}$ points in the second case of $2(d-2)(d-1)$ points.

Let $f_{1}, f_{2}, f_{3}, f_{4}$ be the generators of $I(\Sigma)$, ordered by degree. Since the points of $\Sigma$ are on $X$ it follows that $f \in\left(f_{1}, f_{2}, f_{3}, f_{4}\right)$. Write $f=\sum h_{i} f_{i}$. Since the points of $\Sigma$ are in $X_{\text {sing }}$ and the $f_{i}$ form a system of local coordinates at each point of $\Sigma$ it follows that $h_{i} \in I(\Sigma)$. In particular $f$ is in the ideal generated by $f_{i} f_{j}$. Such a product is of degree at most $d$ only if one of $i, j$ is at most 2. Hence $f \in\left(f_{1}, f_{2}\right)$ and therefore $X$ contains either a plane or a quadric surface, depending on the multidegree of the complete intersection. 
Remark 5.6 This result also implies Theorem 4.1. However, in the above proof we used results from [21], which we can avoid in the proof of Theorem 4.1.

Funding Open access funding provided by Universitá degli Studi di Padova within the CRUI-CARE Agreement.Open access funding provided by Universitá degli Studi di Padova within the CRUI-CARE Agreement.

Open Access This article is licensed under a Creative Commons Attribution 4.0 International License, which permits use, sharing, adaptation, distribution and reproduction in any medium or format, as long as you give appropriate credit to the original author(s) and the source, provide a link to the Creative Commons licence, and indicate if changes were made. The images or other third party material in this article are included in the article's Creative Commons licence, unless indicated otherwise in a credit line to the material. If material is not included in the article's Creative Commons licence and your intended use is not permitted by statutory regulation or exceeds the permitted use, you will need to obtain permission directly from the copyright holder. To view a copy of this licence, visit http://creativecommons.org/licenses/by/4.0/.

\section{References}

1. Cheltsov, I.: Points in projective spaces and applications. J. Differ. Geom. 81, 575-599 (2009)

2. Cheltsov, I.: Factorial threefold hypersurfaces. J. Algebraic Geom. 19, 781-791 (2010)

3. Cheltsov, I.A.: On a conjecture of Ciliberto. Sb. Math. 201, 1069-1090 (2010)

4. Cheltsov, Ivan: Nonrational nodal quartic threefolds. Pac. J. Math. 226(1), 65-81 (2006)

5. Ciliberto, C., Di Gennaro, V.: Factoriality of certain hypersurfaces of $\mathbb{P}^{4}$ with ordinary double points. In Algebraic transformation groups and algebraic varieties, volume 132 of Encyclopaedia Math. Sci., pages 1-7. Springer, Berlin, (2004)

6. Dimca, A.: Betti numbers of hypersurfaces and defects of linear systems. Duke Math. J. 60, 285-298 (1990)

7. Dimca, A.: Singularities and topology of hypersurfaces. Universitext. Springer-Verlag, New York (1992)

8. Eisenbud, D., Green, M., Harris, J.: Higher Castelnuovo theory. Astérisque, 218:187-202, 1993. Journées de Géométrie Algébrique d'Orsay (Orsay, 1992)

9. Finkelnberg, H., Werner, J.: Small resolutions of nodal cubic threefolds. Nederl. Akad. Wetensch. Indag. Math. 51(2), 185-198 (1989)

10. Gotzmann, G.: Eine Bedingung für die Flachheit und das Hilbertpolynom eines graduierten Ringes. Math. Z. 158, 61-70 (1978)

11. Green, M.: Restrictions of linear series to hyperplanes, and some results of Macaulay and Gotzmann. In Algebraic curves and projective geometry (Trento, 1988), volume 1389 of Lecture Notes in Math., pages 76-86. Springer, Berlin, (1989)

12. Green, M.L.: Components of maximal dimension in the Noether-Lefschetz locus. J. Differ. Geom. 29, 295-302 (1989)

13. Greuel, G.-M., Karras, U.: Families of varieties with prescribed singularities. Comp. Math. 69, 83-100 (1989)

14. Hong, K.: Nonrationality of nodal quartic threefolds. Pac. J. Math. 266(1), 31-42 (2013)

15. Kloosterman, R.: Cuspidal plane curves, syzygies and a bound on the MW-rank. J. Algebra 375, 216-234 (2013)

16. Macaulay, F.S.: Some properties of enumeration in the theory of modular systems. Proc. Lond. Math. Soc. S2-26, 531 (1927)

17. Otwinowska, A.: Sur la fonction de Hilbert des algèbres graduées de dimension 0. J. Reine Angew. Math. 545, 97-119 (2002)

18. Otwinowska, A.: Composantes de petite codimension du lieu de Noether-Lefschetz: un argument asymptotique en faveur de la conjecture de Hodge pour les hypersurfaces. J. Algebraic Geom. 12, 307-320 (2003)

19. Peters, C. A. M., Steenbrink, J. H. M.: Mixed Hodge structures, volume 52 of Ergebnisse der Mathematik und ihrer Grenzgebiete. 3. Folge. Springer-Verlag, Berlin, (2008)

20. Shramov, K.A.: $\mathbb{Q}$-factorial quartic threefolds. Mat. Sb. 198(8), 103-114 (2007)

21. Voisin, C.: Composantes de petite codimension du lieu de Noether-Lefschetz. Comment. Math. Helv. 64, 515-526 (1989) 
Publisher's Note Springer Nature remains neutral with regard to jurisdictional claims in published maps and institutional affiliations. 